\title{
Comparative in-vitro Antibacterial Efficacy of the Different Common Herbs and Antibiotics against E.coli and S.aureus Isolated from Bovine Mastitis
}

\author{
Ravi Sikrodia $^{1 *}$, Daljeet Chhabra ${ }^{1}$, Rakhi Gangil ${ }^{1}$, S.D. Audarya ${ }^{1}$, \\ Rakesh Sharda ${ }^{1}$ and S.S. Mahor ${ }^{2}$ \\ ${ }^{1}$ Veterinary Microbiology, COVs \& AH, Mhow, India \\ ${ }^{2}$ Veterinary gynecology and obstetrics, COVs \& AH, Mhow, India \\ *Corresponding author
}

\section{Keywords \\ Herbs, Antibacterial effect, Mastitis, E.coli, S.aureus \\ Article Info \\ Accepted: \\ 08 January 2020 \\ Available Online: \\ 10 February 2020}

A B S T R A C T

Mastitis is the inflammation of the mammary gland and udder tissue, and is a major endemic disease of dairy cattle. It is also the most costly disease to the dairy industry. Bovine mastitis caused by Escherichia coli (E.coli) and Staphylococcus aureus can ranges from being a subclinical infection of the mammary gland to a severe systemic disease. Antimicrobial resistance is generally not a limiting factor for treatment, but it should be monitored to detect changes in resistance profiles. This increases the importance of substitute among antimicrobial agents that kill or inhibit the growth of microorganisms. Some herbs like neem, turmeric, garlic and tulsi have some bioactive molecules that show antibacterial activity. These agents protect the host from cellular oxidation reactions. In the present sudy, in vitro antimicrobial sensitivity of ethanolic extracts of neem, turmeric, garlic and tulsi leaves have been compared with the different antibiotics (ciprofloxacin, ofloxacin and amoxicillin) against E.coli and $S$. aureus isolated from the milk samples of bovine suffering with mastitis. The zone of inhibition of different herbal discs and antibiotics against E.coli and S.aureus were seen after incubation of overnight and measured in millimeter. The maximum zone of inhibition among extracts of herbs and antibiotic were found in neem for E.coli followed by turmeric, ofloxacin and ciprofloxacin. The extracts of tulsi, garlic and antibiotic amoxicillin were found resistant against E.coli. While in case of S.aureus the maximum zone was found in ciprofloxacin followed by tulsi, ofloxacin, garlic, neem and amoxicillin. The extract of turmeric was not showing any sensitivity against S.aureus.

\section{Introduction}

Mastitis is the inflammation of the mammary gland and udder tissue, and is a major endemic disease of dairy cattle. It is also the most costly disease to the dairy industry. Bovine mastitis caused by Escherichia coli
(E.coli) can range from being a subclinical infection of the mammary gland to a severe systemic disease. Cow-dependent factors such as lactation stage and age affect the severity of coliform mastitis. Evidence for the efficacy of antimicrobial treatment for $E$. coli mastitis is very limited. Another bacteria 
Staphylococcus aureus is also one of the most common types of chronic mastitis. Though some cows may flare up with clinical mastitis (especially after calving) the infection is usually subclinical, causing elevated somatic cell counts (SCC) but no detectable changes in milk or the udder. (Tenhagen et al., 2007)

Antimicrobial resistance is generally not a limiting factor for treatment, but it should be monitored to detect changes in resistance profiles. Injudious and rampant use of different antibiotics now increases the resistance in microbes. (Suojala et al., 2013).

This increases the importance of substitute among antimicrobial agents that kill or inhibit the growth of microorganisms. Some herbs like neem, turmeric, garlic and tulsi have some bioactive molecules that show antibacterial activity. These agents protect the host from cellular oxidation reactions.

Tulsi (Ocimum tenuiflorum synonym Ocimum sanctum) commonly known as holy basil or tulasi is an aromatic perennial plant in the family Lamiaceae. It is a shrub having oleanolic acid, ursolic acid, rosamarinic acid, eugenol, carvacrol, linalool, $\beta$ elemene, germacrene as chemical constituents that have antimicrobial activity (Warrier, 1995).

Turmeric is a flowering plant, Curcuma longa of the ginger family, Zingiberaceae, the roots of which are used in cooking. Curcumin is a bright yellow chemical produced by Curcuma longa plants. Curcumin has been used historically in Ayurvedic medicine. (Priyadarsini, 2014)

Neem (Azadirachta indica) commonly known as nimtree or Indian lilac is a tree in the mahogany family Meliaceae. It is one of two species in the genus Azadirachta, and is native to the Indian subcontinent. Neem is a fast growing evergreen tree having $20-40 \mathrm{~cm}$ long leaves. It has acetic acid, hydroxy piralic, phytol, 4- cyclooctenol, 1, 3 diphenyl2-azafluorene, acetate, germnicol (Alzohairy, 2016). Garlic (Allium sativum) is a species in the family Alliaceae. Active principle allicin is an organosulfur compound obtained from garlic and showing antibacterial and antifungal efficacy. (Marchese 2016)

In the present sudy, in vitro antimicrobial activity of the ethanolic extracts of neem, turmeric, garlic and tulsi leaves have been compared with the different antibiotics against E.coli and S. aureus isolated from the milk samples of bovine suffering with mastitis.

\section{Methodology}

The present research work was carried out in the laboratory of the department of Veterinary Microbiology, College of Veterinary Science and Animal Husbandry, Mhow, Indore (M.P.).

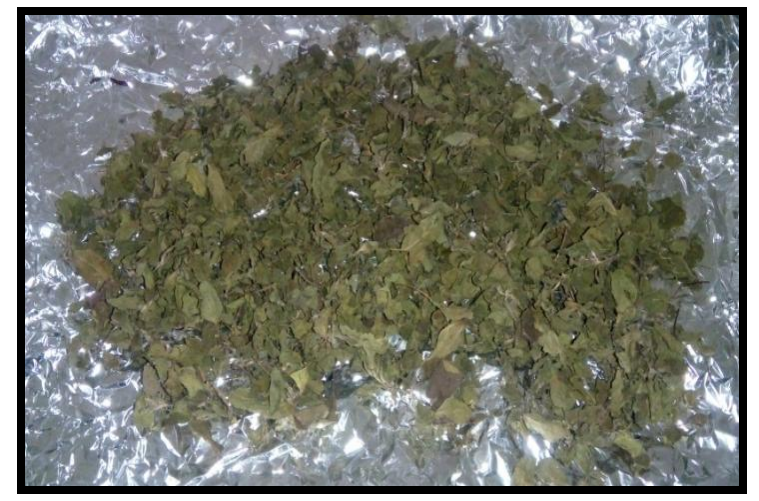

Fig.1 Tulsi leaves

\section{Preparation of different herbal extracts}

The leaves of the Tulsi (Fig 1) and Neem plants (Fig 3) were washed to clean the dirt and dried in shadow and then grinded to form powder and kept in air tight bottle. The garlic bulb (Fig 2) was pealed, washed, dried and grinded to make powder kept in the airtight bottle. Dried rhizome of turmeric (Fig 4) was 
grinded to make powder and kept in airtight bottle. These powders were filled up in the filter paper thimble and then thimbles were fixed in the soxlet extraction assembly.

Extraction was carried out with ethanol at $80^{\circ} \mathrm{c}$ temperature. The extract was recovered and extra ethanol was evaporated (Fig 5). These extracts were used to prepare the disc for the in-vitro antibacterial sensitivity test.

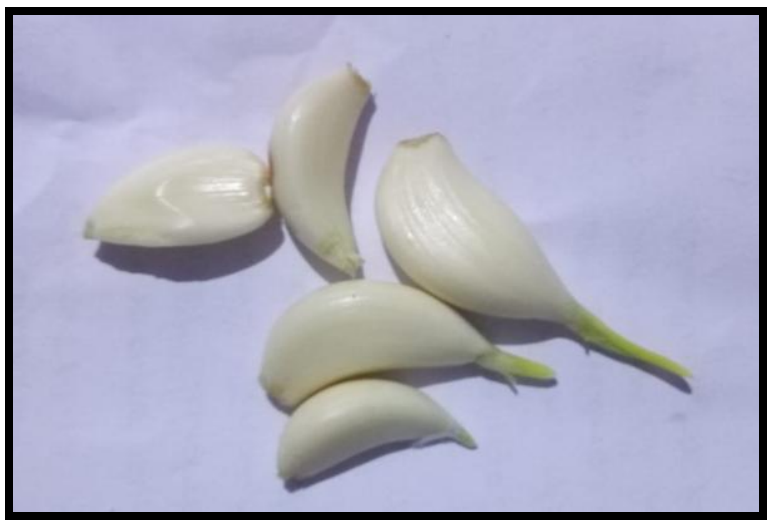

Fig.2 Garlic bulbs

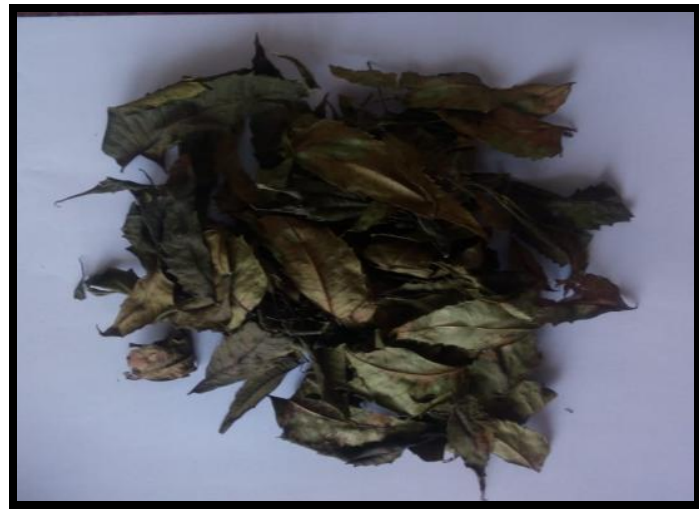

Fig.3 Neem leaves

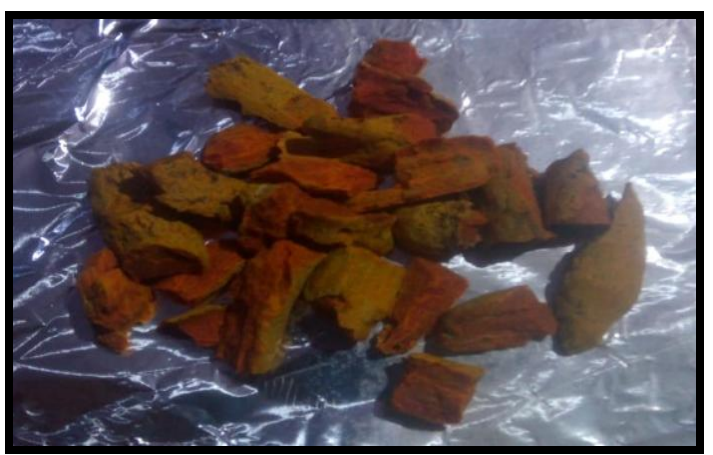

Fig.4 Turmeric bud

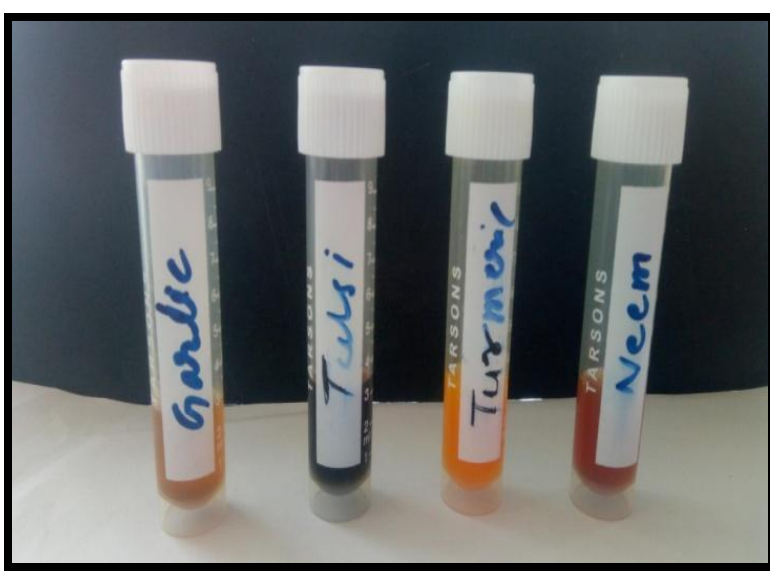

Fig.5 Different ethanolic extracts of Garlic, Tulsi, Turmeric and Neem

\section{Antibiotic discs}

The readymade ciprofloxacin, ofloxacin and gentamycin antibiotic discs (Himedia, Fig 6) were used in the study to compare antibacterial efficacy with different herbal disc.

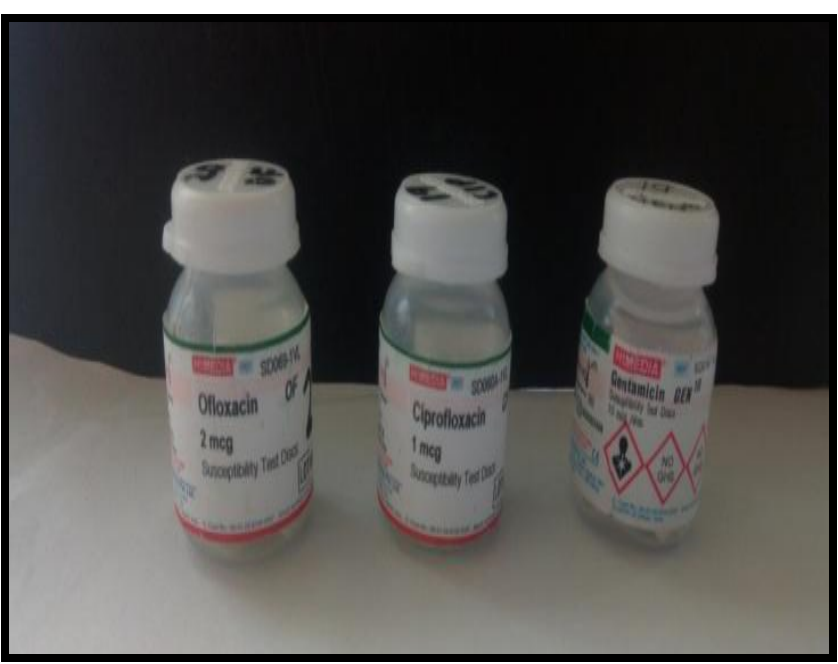

Fig.6 Different antibiotics used in in-vitro antibacterial sensitivity test

\section{Preparation of disc from herbal extracts}

The sterile discs (Himedia, Fig 7) were used in present study. The disc were soaked in the extracts for overnight and dried in incubator at $50^{\circ} \mathrm{c}$ for overnight. Dried disc were used in the in-vitro antibacterial sensitivity test 


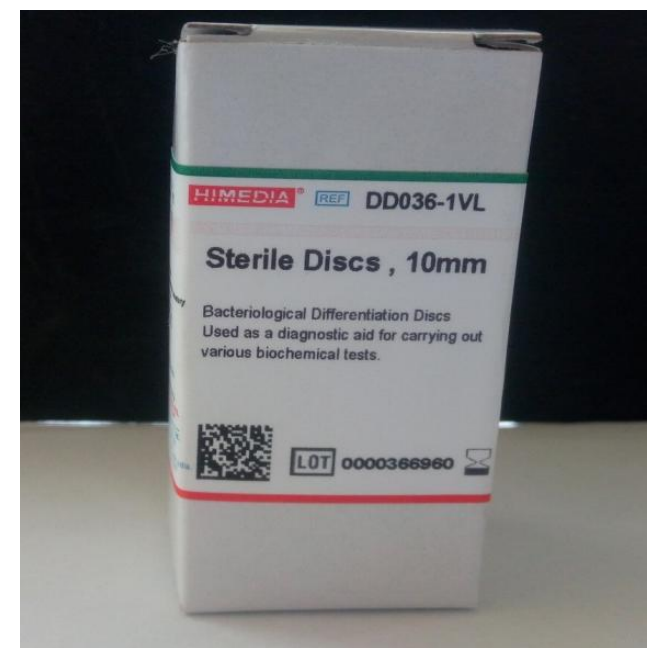

Fig.7 Sterile discs used in preparation of herbal disc

\section{Preparation of media}

In the present study BHI (Brain Heart Infusion) broth (M210-500G, Himedia), Nutrient agar, EMB agar (Himedia), Baired parker agar (Himedia) and MHA (Muellar Hinton Agar) media (M173-500G, Himedia) were used. The media was prepared by mixing the powder in distle water as per the specification given by the manufacturer. After proper mixing it was sterilized by autoclaving at pressure of $15 \mathrm{lbs}$ temperature $121^{\circ} \mathrm{c}$ for 15 minuts. Then poured in petriplates for further use in the study.

\section{Test organisms}

The E. coli and S. aureus were isolated from the different milk samples positive for the CMT (California Mastitis test). The samples were found positive for the CMT inoculated in the BHI broth for overnight.

The nutrient agar plates were inoculated from the BHI and incubated at $37^{\circ} \mathrm{c}$ for $24 \mathrm{hrs}$. then the single colony from the nutrient agar plates were taken and streaked on the selected media EMB (Eosine methylene Blue) agar and baired parker agar and then incubated at $37^{\circ} \mathrm{c}$ for overnight. The E. coli organisms showing metallic sheen on EMB agar while the $S$. aureus showing black colnies with halo pattern on Baired parker agar were transferred on nutrient agar slant and stored for the invitro sensitivity test.

\section{Disc diffusion method}

For the invitro sensitivity testing the isolated E.coli and $S$. aureus from nutrient agar slants were inoculated in BHI broth and incubated at $37^{\circ} \mathrm{c}$ for $4-5 \mathrm{hrs}$. Then with a sterile swab prepare a lawn of E.coli and S.aureus culture on the different MHA plates. All the prepared herbal discs along with antibiotic disc were aseptically placed on agar surfaces. These plates were incubated at $37^{\circ} \mathrm{c}$ for overnight.

\section{Results and Discussion}

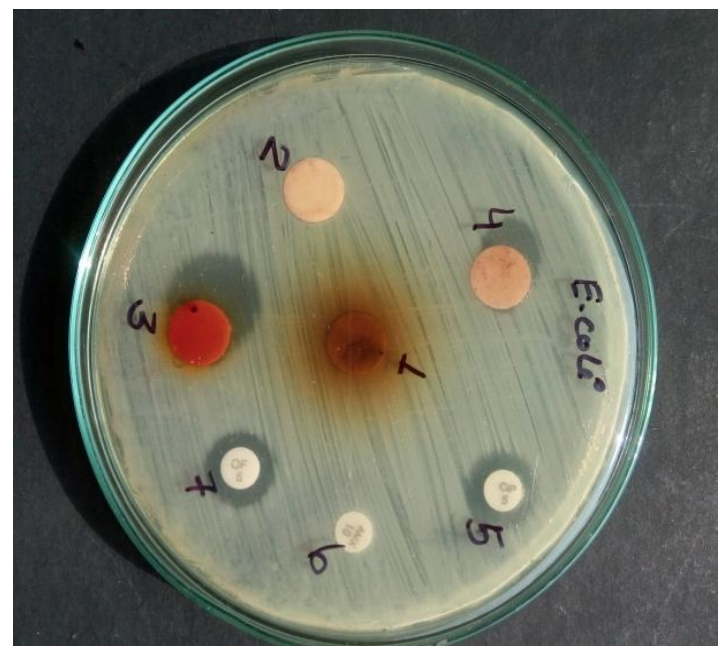

Plate.1 Zone of inhibition of different herbal discs and antibiotics against E.coli

The zone of inhibition of different herbal discs and antibiotics against E.coli (Plate 1) and S.aureus (Plate 2) were seen after incubation of overnight and measured in millimeter shown in table 1. All ethanolic extracts of herbs and antibiotics showed the antimicrobial property. The extract of tulsi showed the zone of inhibition (ZOI) against the S.aureus $(22 \mathrm{~mm})$ while found resistant (no zone) against the E.coli. Neem extract 
was showed zone of inhibition against both E.coli and S.aureus $16 \mathrm{~mm}$ and $19 \mathrm{~mm}$ respectively. Turmeric was found sensitive against E.coli $14 \mathrm{~mm}$ and found resistant against S.aureus. Garlic extrac was found resistant against E.coli and sensitive against S.aureus $(20 \mathrm{~mm})$. The antibiotic discs (Himedia) of ciproflaoxacin, ofloxacin and amoxicillin were found sensitive against both E.coli and S.aureus.

These discs of antibiotics were showed zone of sensitivity 11 and $24 \mathrm{~mm}$ for ciprofloxacin, 12 and 21 for ofloxacin and 0 and 14 for amoxicillin against E.coli and S.aureus respectively. The maximum zone of inhibition among extracts of herbs and antibiotic were found in neem for E.coli followed by turmeric, ofloxacin and ciprofloxacin.

The extracts of tulsi, garlic and antibiotic amoxicillin were found resistant against E.coli. While in case of S.aureus the maximum zone was found in ciprofloxacin followed by tulsi, ofloxacin, garlic, neem and amoxicillin. The extract of turmeric was not showing any sensitivity against S.aureus.

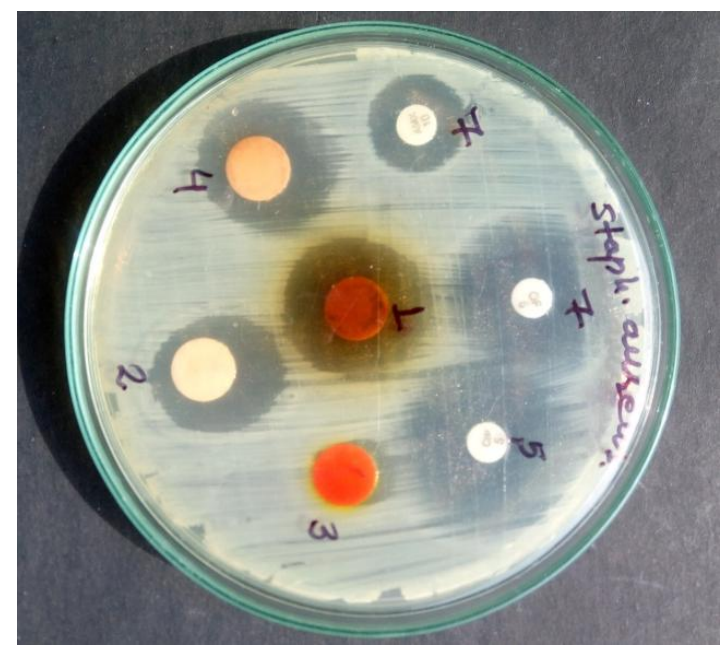

Plate.2 Zone of inhibition of different herbal discs and antibiotics against S.aureus

Table.1 Zone of inhibition of different herbal extracts and antibiotics against E.coli and S.aureus

\begin{tabular}{|l|l|c|c|}
\hline S.N. & Disc & Zone of inhibition (millimeter) \\
\hline & & E.coli & S. aureus \\
\hline $\mathbf{1}$ & Tulsi & 0 & 22 \\
\hline $\mathbf{2}$ & Neem & 16 & 19 \\
\hline $\mathbf{3}$ & Turmeric & 14 & 0 \\
\hline $\mathbf{4}$ & Garlic & 0 & 20 \\
\hline $\mathbf{5}$ & Ciprofloxacin & 11 & 24 \\
\hline $\mathbf{6}$ & Ofloxacin & 12 & 21 \\
\hline $\mathbf{7}$ & Amoxicillin & 0 & 14 \\
\hline
\end{tabular}




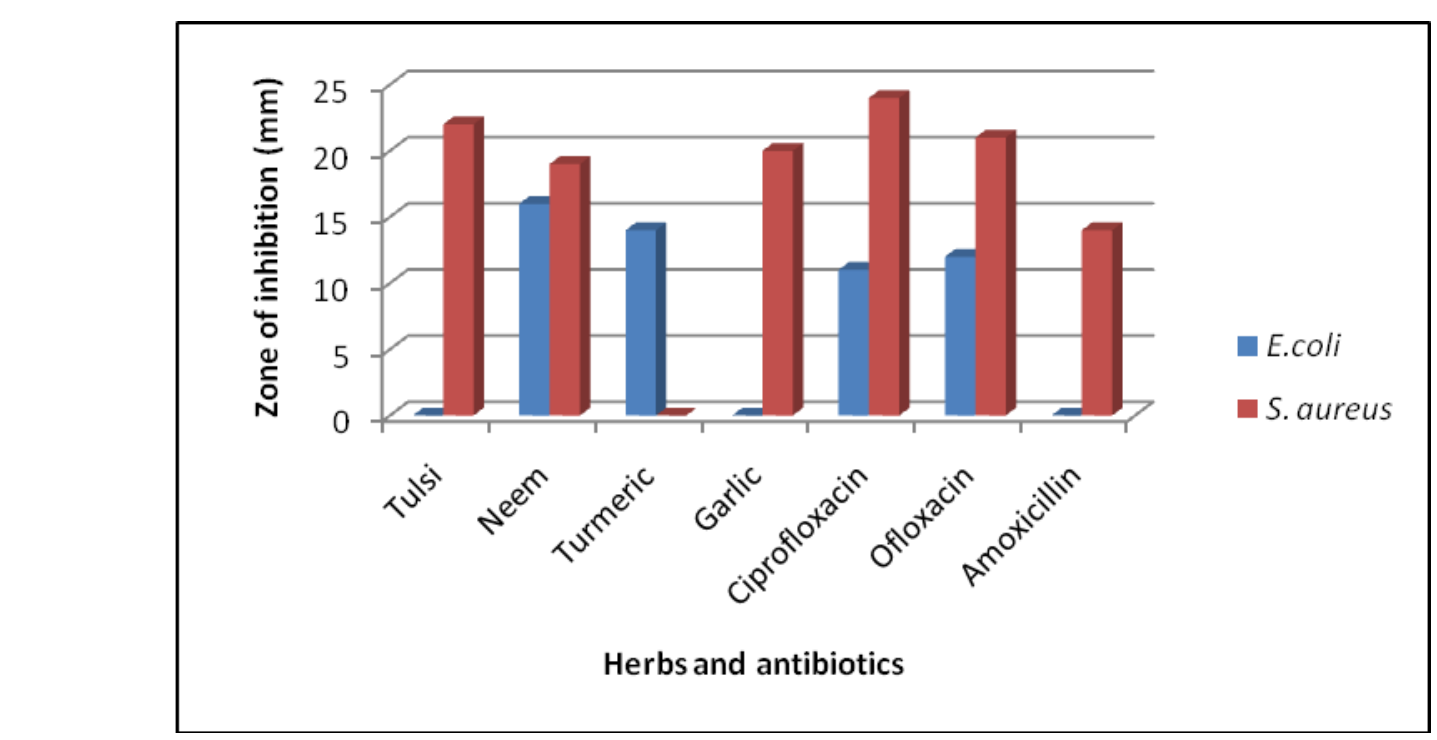

Graph.1 Comparitive zone of inhibition of different herbal discs and antibiotics against E.coli and S.aureus

Mastitis is an important multietiological disease problem affects major population of animals causes heavy economical loss. The treatment is becoming difficult due to emergence of multidrug resistance amongst pathogens.

The organisms included in the study are two major multidrug resistant pathogens involved in the mastitis in large animals. It is therefore necessary to search the natural compounds shows potent antimicrobial property. Many herbal plants are drawing increasing attention all over the world.

The herbal plants investigated in the present study showed good antimicrobial potential against gram positive (S.aureus) and gram negative (E.coli) bacteria. Many herbal plants were showed better antimicrobial efficacy than the antibiotics used in the study may be due to development of resistance against these antibiotics.

Thus, after entire study it can be concluded that in herbal plants tulsi have maximum antibacterial efficacy against S.aureus while neem have maximum efficacy against E.coli. Thus the ethanolic extracts of these plants can be further investigated to identify the potential antimicrobial compounds present which can serve as important candidate for drug formation.

\section{References}

Alzohairy, M.A. (2016). Therapeutics role of Azadirachta indica (Neem) and their active constituents in diseases prevention and treatment. Evid Based Complement Altern Med. 7382506.

Kumar, V., Chakraborty, A., Kaur, M., Pandey, S. and Jena, M.K. (2018). comparative study on antimicrobial activity of tulsi (ocimum sanctum) and neem (azadirachta indica) methanol extract. 11:12.

Marchese, A., Barbieri, R., Sanches-Silva, A., Daglia, M., Nabavi, S.F., Jafari, N.J., Izadi, M., Ajami, M. and Nabavi, S.M. (2016). "Antifungal and antibacterial activities of allicin: A review". Trends in Food Science and Technology. 52: 49.

Mariana, C., Henrique, M.C., Massuco, J., Tais, M.B. and Luis, V. S. (2017). Phytochemical screening of Azadirachta indica A. Juss for 
antimicrobial activity. Afr J Microbiol Res. 11:117-22.

Mukhtar, S. and Ghori, I. (2010). Antibacterial activity of aqueous and ethanolic extracts of garlic, cinnamon and turmeric against Escherichia coli ATCC25922 and Bacillus subtilus DSM 3256. International journal of applied biology and pharmaceutical technology, 3.

Priyadarsini, K.I. (2014). "The chemistry of curcumin: from extraction to therapeutic agent," Molecules. 19:2091-20112.

Suojala, L., Kaartinen, L. and Pyorala, S. (2013). Treatment for bovine Escherichia coli mastitis - an evidence- based approach. J. Vet. Pharmacol. Ther. 36(6):521-31.

Tenhagen, B. A., I. Hansen, A. Reinecke, and W. Heuwieser. 2009. Prevalence of pathogens in milk samples of dairy cows with clinical mastitis and in heifers at first parturition. Journal of Dairy Research 76(2): 179-87.

Warrier, P. K. (1995). Indian medicinal plants. OrientLongman.

Yamani, H.A., Pang, E.C., Mantri, N. and Deighton, M.A. (2016). Antimicrobial activity of tulsi (Ocimum tenuiflorum) essential oil and their major constituents against three species of bacteria. Front Microbiol. 7:681.

\section{How to cite this article:}

Ravi Sikrodia, Daljeet Chhabra, Rakhi Gangil, S.D. Audarya, Rakesh Sharda and Mahor. S.S. 2020. Comparative in-vitro Antibacterial Efficacy of the Different Common Herbs and Antibiotics against E.coli and S.aureus Isolated from Bovine Mastitis. Int.J.Curr.Microbiol.App.Sci. 9(02): 1069-1075. doi: https://doi.org/10.20546/ijcmas.2020.902.125 